\title{
A New Concept: A Peptide Monolayer for Accurate Positioning of Electroactive Probes at Close Distance from a Gold Surface
}

\author{
M. Caruso ${ }^{1}$, E. Gatto ${ }^{1}$, A. Palleschi ${ }^{1}$, M. Scarselli ${ }^{2}$, M. De Crescenzi ${ }^{2}$, \\ K. Wright ${ }^{3}$, E. Longo ${ }^{4}$, M. De Zotti ${ }^{4}$, F. Formaggio ${ }^{4}$, C. Toniolo ${ }^{4}$, \\ and M. Venanzi ${ }^{1}$ \\ ${ }^{1}$ Department of Chemical Sciences and Technologies, University of Rome 'Tor Vergata', 00133, Rome, Italy; \\ ${ }^{2}$ Department of Physics, University of Rome 'Tor Vergata', 00133, Rome, Italy; ${ }^{3}$ Institute \\ Lavoisier de Versailles, UMR 8180, University of Versailles, 78035, Versailles, France; \\ ${ }^{4}$ Department of Chemistry, University of Padova, 35131, Padova, Italy
}

\section{Introduction}

Thio-gold chemistry is the most extensively employed method to generate organic layers stably linked to gold. Peptide functionalization at the $N$ - and/or $C$-terminus with thiol linkers has been widely utilized, as well as with the side chains of sulfur containing proteinogenic amino acids (as Cys or Met). Among the several linkers to gold, dithiolane (with an S-S bridge) has been exploited for its ability to induce strong bidentate ligation. In this connection, lipoic acid has been the most popular compound for realizing this kind of linkage. At the beginning of the 1970s, a family of new dithiolane-containing cyclic structures, and among them the 4-amino-1,2-dithiolane-4-carboxylic acid (herein called Adt), was originally synthesized as an organic building block. Belonging to the class of $\mathrm{C}^{\alpha, \alpha}$-cyclized, $\mathrm{C}^{\alpha}$-tetrasubstituted $\alpha$-amino acids [1], Adt is expected to stabilize 310 -helical structures in short peptides.

Beside its unique conformational properties, the presence of an S-S dithiolane group on the side chain ring makes Adt the best candidate for developing a new approach to peptide functionalization of gold surfaces. The short separation of the S-S linker from the peptide backbone allows the design of peptide layers much closer to the metal surface as compared to those formed by peptides using lipoic acid as the linker. Furthermore, bidentate ligation should anchor the peptide chain to the gold surface more rigidly than would a flexible linker like Cys. We therefore synthesized a hexapeptide, denoted in the following as Fc-CO-6Adt2, formed by two -Adt-Ala-Aib- triads and functionalized with an electroactive ferrocenoyl $(\mathrm{Fc})$ group at the $\mathrm{N}$-terminus [ $\mathrm{Fc}-\mathrm{CO}$-(Adt-Ala-Aib) ${ }_{2}-\mathrm{OMe}$. The presence in the oligopeptide sequence of four $\mathrm{C}^{\alpha}$-tetrasubstituted residues, i.e. the two Adt and the two Aib residues, gives a strong propensity to the main chain to attain a 310 -helix conformation both in solution and in the crystal state, and consequently to locate the two Adt residues in the correct positions to link to the gold surface.

\section{Results and Discussion}

The predominant population of a 310 -helical conformation in Fc-CO-6Adt 2 was confirmed by FTIR absorption and 2D-NMR (NOESY, TOCSY) experiments in solution and by X-ray diffraction crystal studies on the synthetic pentapeptide precursor. X-Ray Photoelectron Spectroscopy (XPS) and Scanning Tunneling Microscopy (STM) techniques were used to characterize the binding of the peptide to the gold surface and the morphology of the peptide layer, respectively. In particular, XPS data indicate separate components for the $S(2 \mathrm{p})$ element: the signals at $161.5 \mathrm{eV}(36 \%)$ and $162.5 \mathrm{eV}$ (4\%) that can be assigned to Au-bonded sulfur atoms (thiolate bonded to the Au hollow sites), and the signal at $163.7(40 \%)$ typical of non-bonded or weakly interacting sulfur atoms. A fourth component at $165.3 \mathrm{eV}(20 \%)$ can be assigned to disulfide groups or oxidized (sulfoxides or sulfones) sulfur atoms. STM measurements on the Fc-CO-6Adt2-modified substrate revealed that extended regions of the gold surface appeared strongly altered by the presence of the peptide layer, which exhibits a rich morphology, i.e. emerging clusters of peptides of nanometric size and ordered stripe-like structures. 

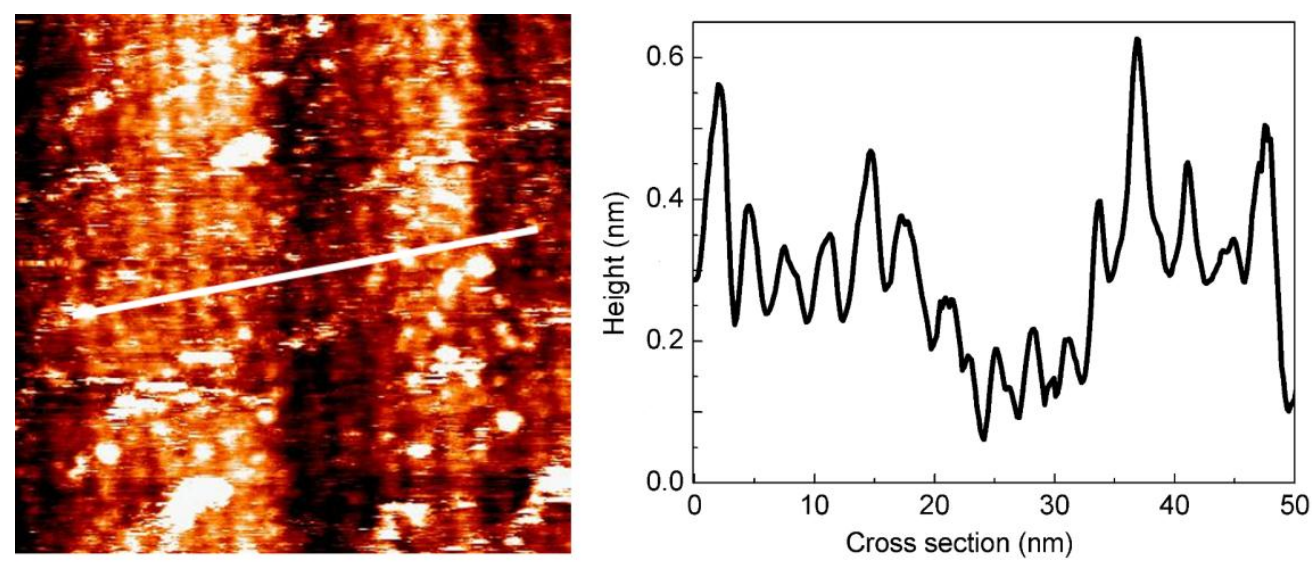

Fig. 1. Left: STM image of a stripe domain of the Fc-CO-6Adt2 film on gold. Bright spots of 1-nm width and 0.5-nm height can also be seen in the image $\left(55 \times 55 \mathrm{~nm}^{2}, V_{s}=1 \mathrm{~V}, I=300 \mathrm{pA}\right)$. Right: Height profile taken along the line drawn in the image reported on the left side.

Interestingly, stripe domains (Figure 1) showed ordered flat structures of 2-3 nm width and sub-nanometric height, i.e. the actual size dimensions of peptides horizontally layered on the gold surface. Cyclic Voltammetry (CV) experiments carried out on the Fc probe showed that the current intensity increases linearly with the $\mathrm{CV}$ scan rate, indicating that the Fc discharge is not affected by diffusive processes at the electrode/peptide interface, i.e. that the peptide is stably chemisorbed on the surface.

Chronoamperometry (CA) experiments were also carried out on a three-electrode electrochemical cell using an Fc-CO-6Adt2-modified gold electrode as the working electrode. We found that CA experiments were adequately reproduced by a bi-exponential decay law, obtaining $k_{0,1(a)}=11.2 \pm 0.4 \mathrm{~s}^{-1}$ under anodic conditions, i.e. ET Fc $\rightarrow \mathrm{Au}$, and $k_{0,1(c)}=12.3 \pm 0.4 \mathrm{~s}^{-1}$ under cathodic conditions, i.e. ET for $\mathrm{Au} \rightarrow \mathrm{Fc}$ for the faster time component of the current intensity decay and $k_{0,2(a)}=0.9 \pm 0.2 \mathrm{~s}^{-1}$ and $k_{0,2(c)}=1.2 \pm 0.3 \mathrm{~s}^{-1}$ under anodic and cathodic conditions, respectively, for the slower time component. The appearance in the plot of frequency-normalized current intensity $v s$. frequency, obtained by pulsed Square Wave Voltammetry (SWV), of a maximum at a critical frequency of about $100 \mathrm{~Hz}$, strongly suggests that most of the Fc probes are rigidly positioned at a relatively close distance from the gold surface.

\section{Conclusions}

In this contribution we described a new strategy for functionalizing a solid substrate with a peptide layer characterized by the main chain being rigidly disposed in a parallel orientation with respect to the surface. This result was achieved by inserting in the peptide sequence two Adt residues in the correct position to realize two bidentate linkages to the gold surface through the two dithiolane sulfur atoms in the Adt side chain. The main achievement of this new strategy of functionalizing solid substrates is to make possible an accurate positioning of an electroactive probe in the proximity of the gold surface. We proved this situation by analyzing by several electrochemical techniques the properties of a Fc group covalently linked to the peptide chain at its $N$-terminus.

\section{Acknowledgments}

This work was supported by the Italian Ministry of University and Research (MIUR), PRIN 2010-2011 No. 2010FM738P, 'Photophysical and photochemical properties of organic and biological compounds in solution and in organized systems'.

\section{References}

1. Toniolo, C., Crisma, M., Formaggio, F., Peggion, C. Biopolymers (Pept. Sci.) 60, 396-419 (2001), http://dx.doi.org/10.1002/1097-0282(2001)60:6<396::AID-BIP10184>3.0.CO;2-7 